\title{
DEUX NOUVELLES ESPĖCES DU GENRE GORGODERA
}

\author{
Par S. V. PIGUlevsky
}

Nous ayons trouvé, dans la vessie urinaire de grenouilles (Rana ridibunda Pallas), provenant de Fachkent, Keles et Syr-Daria, de l'Asie moyenne, deux espèces nouvelles de Gorgodéridés.

\section{Gorgodera asiatica nov, spec. (fig. 1)}

Ce trématode, de forme lancéolée, a une longueur de $4 \mathrm{~mm}$, 8-6 $\mathrm{mm}$. et une largeur maxima de $1 \mathrm{~mm}$., 14-1 mm., 65. La ventouse buccale a $330-450 \mu$ de diametre. La ventouse ventrale, 510$750 \mu$ de diamètre. L'osophage est relativement court, 90-180 $\mu$ de longueur ; les deux branches de l'intestin n'atteignent pas l'extrémité postérieure du corps. Les testicules ont des contours irrégulièrement lobés; ils sont au nombre de $7(3+4)$ ou de $9(4+5)$. Le plus grand testicule mesure $240-300 \mu \times 300-330 \mu$ et le plus petit $150-180 \mu \times 210-230 \mu$. La glande vitellogène est en forme de grappe de raisin, à lobes ovales, au nombre de 7 de chaque côté. L'ovaire, presque sphérique et non lobé, est situé du côté gauche de la ligne médiane, et mesure $360 \mu$ de largeur sur $390 \mu$ de longueur. Les organes génitaux sont très rapprochés du milieu du corps. L'utérus est très fortement ondulé, remplissant tout l'espace libre en arrrière des glandes génitales. Les œufs, mesurés près du pore génital, ont $17-23 \mu$ sur $23-29 \mu$.

\section{Gorgodera dollfusi nov. spiec. (fig. 2)}

Le ver adulte est fusiforme avec une longueur de 2 mm., 85; sa plus grande largeur est à la hauteur de la puissante ventouse ventrale, où elle atteint $660 \mu$. La ventouse buccale a $300 \mu$ de large sur $330 \mu$ de long. La ventouse ventrale, ovale, a $390 \mu$ de large sur $420 \mu$ de long. L'oxsophage est très court, $90 \mu$ de long. Les deux branches du tube digestif se terminent dans la partie postérieure du corps. L'intestin présente de petils diverticules; il se

Axx. ve L'ARASITOLOGe, T, XX, $x^{* 0} 5-6,1944-1945$, p. 28t-287. 
bifurque juste en avant de la ventouse ventrale. Les testicules sont lobulés et placés asymétriquement. Quatre testicules, situés du côté droil, et cinq du côté gauche. Le plus grand testicule mesure

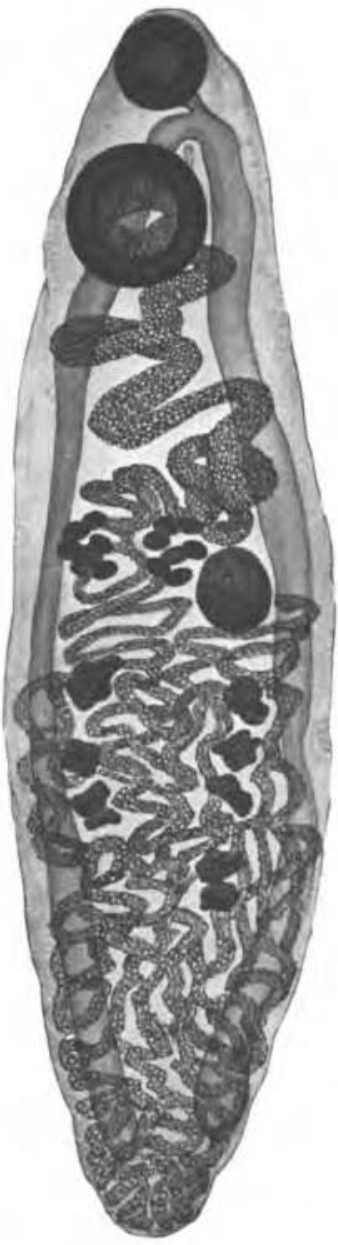

Fig. 1. - Gorgodera

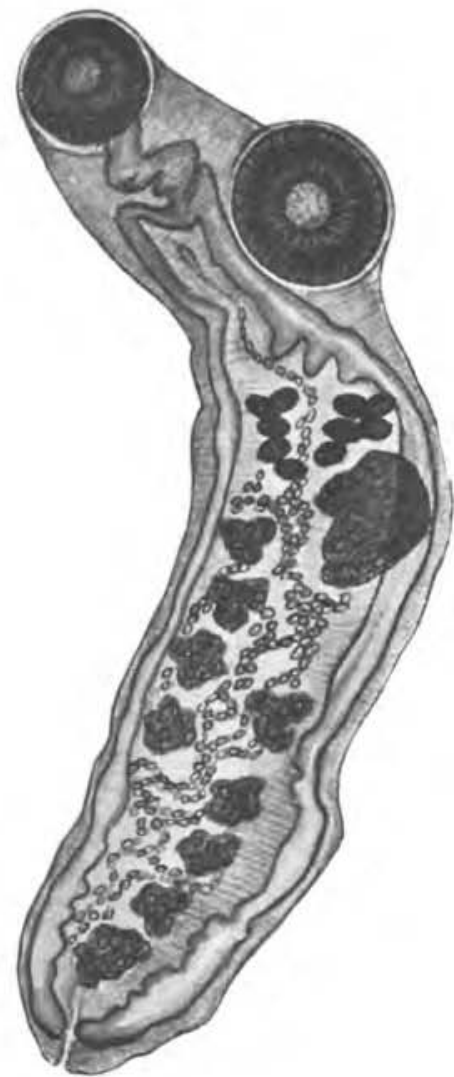

Fig. 2. - Gorgoderd dollfusi n. sp. asiatica n. sp.

$150 \mu$ de large el $210 \mu$ de long, et le plus petit $120 \mu \times 180 \mu$. Lá glande vitellogène est en forme de grappe de raisin, avec des lobes ovales au nombre de 5 de chaque côté. Elle ast disposée obliquement du còté gauche du corps, entre l'ovaire et la ventouse ven- 


\begin{tabular}{|c|c|c|c|c|c|c|c|c|c|c|c|c|}
\hline 空 & $\begin{array}{l}\text { ปิ } \\
\times \\
\text { वें }\end{array}$ & & $\begin{array}{l}\stackrel{\infty}{*} \\
\times \\
0 \\
0\end{array}$ & 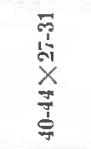 & $\begin{array}{l}\equiv \\
x \\
\infty\end{array}$ & 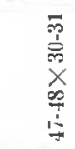 & $\begin{array}{l}\stackrel{\sim}{N} \\
\times \\
\equiv\end{array}$ & $\begin{array}{l}\text { si } \\
\times \\
\text { iो }\end{array}$ & 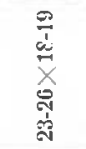 & 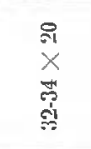 & 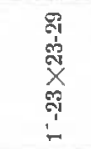 & 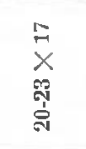 \\
\hline 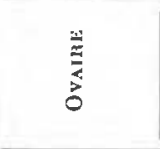 & 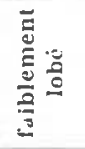 & $\stackrel{\circ}{0}$ & $\frac{\stackrel{n}{\stackrel{D}{E}}}{\infty}$ & 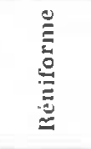 & :ัّ & 节 & 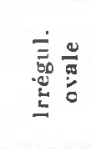 & $\stackrel{\leftrightarrows}{\leftrightarrows}$ & 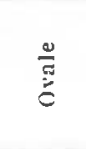 & $\frac{c}{E}$ & 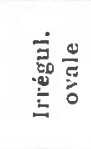 & 过 \\
\hline 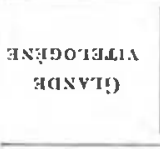 & 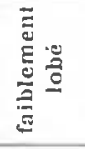 & 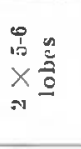 & $\begin{array}{l}\infty \\
0 \\
0 \\
\times \\
\times \\
0 \\
\infty 1\end{array}$ & $\begin{array}{l}\infty \\
\stackrel{0}{0} \\
\times \stackrel{0}{0} \\
-1\end{array}$ & 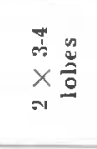 & 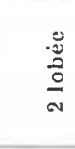 & 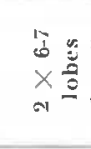 & $\begin{array}{l}11 \\
\times \stackrel{n}{0} \\
\sigma_{1}\end{array}$ & $\begin{array}{l}-0 \\
\times \\
\text { N }\end{array}$ & 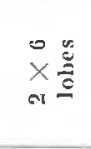 & $\begin{array}{l}1 \\
\times \\
N \\
N\end{array}$ & $\begin{array}{l}100 \\
\times \frac{0}{0} \\
\text { N }\end{array}$ \\
\hline SATIOILS:I.J & \multicolumn{4}{|c|}{ 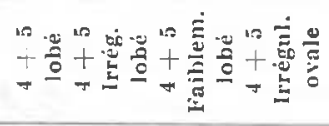 } & $\begin{array}{l}10 \\
+0 \\
+0\end{array}$ & \multicolumn{4}{|c|}{ 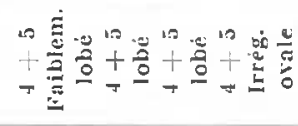 } & 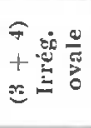 & 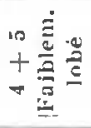 & 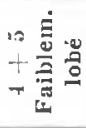 \\
\hline 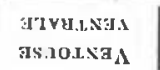 & 品 & 㛭 & 일 & 瓷尽 & 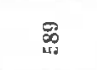 & & & & Е ํㅗำ & : & 初是 & 突荨 \\
\hline 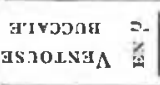 & ํํำ & 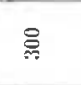 & 品品 & ถิำ ปี่ & 席 & & & & 气总 & 유 & 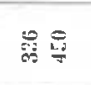 & 䎏 윰 \\
\hline MaISUV' & & $\stackrel{19}{5}$ & 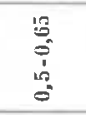 & $\stackrel{\square}{\vdots}$ & $\stackrel{2}{2}$ & - & & & $\stackrel{0}{=}$ & $\because$ & 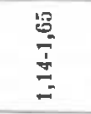 & $\stackrel{8}{8}$ \\
\hline 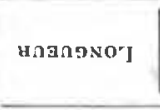 & 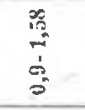 & $\because$ & 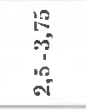 & 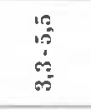 & is & $\stackrel{9}{\stackrel{2}{\circ}}$ & & & $\therefore$ & 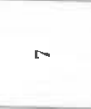 & $\dot{1}$ & $\begin{array}{l}2 \\
-i\end{array}$ \\
\hline 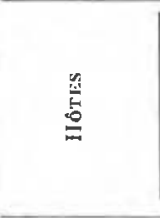 & 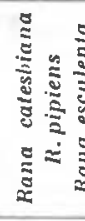 & 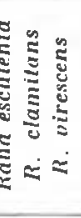 & 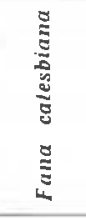 & 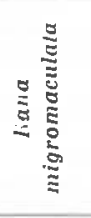 & 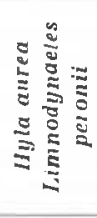 & 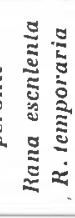 & 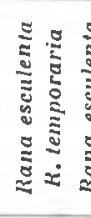 & 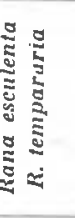 & 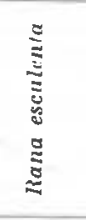 & 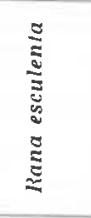 & 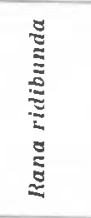 & 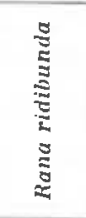 \\
\hline 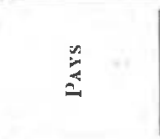 & 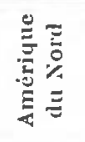 & $=$ & $\approx$ & ڤ్ & $\frac{\triangleq}{\bar{E}}$ & 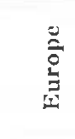 & $\begin{array}{l}= \\
=\end{array}$ & $=$ & $\begin{array}{l}\hat{\theta} \\
\end{array}$ & $\approx$ & $\frac{1}{3}$ & $\stackrel{n}{4}$ \\
\hline במY & $\frac{\Omega 1}{5}$ & $\stackrel{g}{\mathscr{g}}$ & ฐิ & 超 & $\stackrel{9}{\Xi}$ & 蒙 & 訚 & 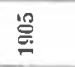 & $\stackrel{\pi}{\leftrightarrows}$ & ड્' & $\overline{\widetilde{g}}$ & 苛 \\
\hline 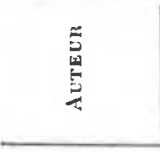 & ¿ & $\stackrel{2}{0}$ & 离 & 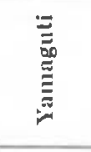 & $\frac{\Xi}{\frac{0}{0}}$ & 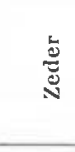 & $\stackrel{\Xi}{\stackrel{\Xi}{N}}$ & 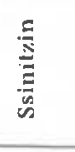 & 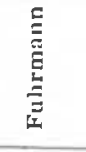 & 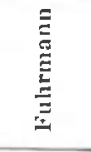 & 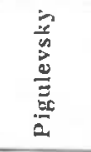 & 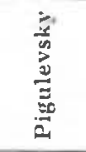 \\
\hline 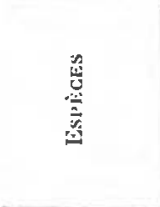 & $\begin{array}{c}\stackrel{\Xi}{\Xi} \\
\stackrel{\Xi}{\Xi} \\
\stackrel{\Xi}{\Xi}\end{array}$ & 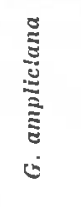 & 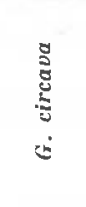 & 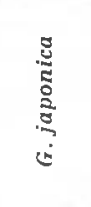 & 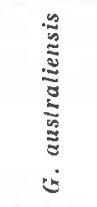 & 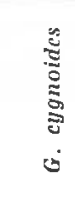 & 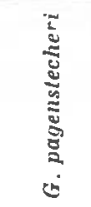 & 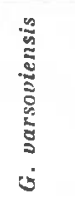 & $\begin{array}{l}0 \\
\stackrel{0}{5} \\
0 \\
\stackrel{0}{0} \\
\vdots \\
0\end{array}$ & 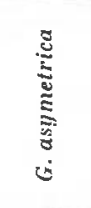 & 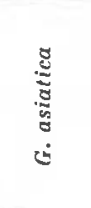 & 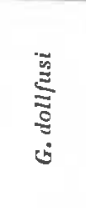 \\
\hline$\therefore$ & $\vdots$ & $\dot{\mathrm{a}}$ & $\dot{m}$ & $\vdots$ & $\therefore$ & : & $\therefore$ & $\infty$ & $\vdots$ & $\dot{0}$ & $\vdots$ & i্ \\
\hline
\end{tabular}


trale. L'ovaire, irrégulièrement lobé, est situé en avant des testicules du côté gauche de la ligne médiane du corps. Les glandes génitales sont dans la partie postérieure du corps. Les sinuosités de l'utérus remplissent l'espace environnant les organes génitaux. Les œufs du parasite conservé ont, près du pore génital, un diamétre longitudinal de 20-23 $\mu$, tandis que le diamètre transversal est de $17 \mu$. Le pore génital est placé au milieu de la partie antérieure.

\section{Bibliographie}

Bensley. - Centralbl, Bakt, XXI, 1897, p. 326-331, fig. 1-2.

ConT (W.). - Erans. Americ. Micr. Soc., XXXI, 1912, p. 151-166, pl. XV, XVI.

Dirsivg (C.). - Systema helminthum, I, 1850, p. 342.

Dujandin (F.). - Histoire naturelle des Helminthes, 1845, p. 396.

Funimane (O.). - Bull. Soc. neuchat. Sc. nat., XI.IX, 1924, p. 131-137, fig. 1-2.

Guberlet (J.). - Frans. Americ. Micr. Soc., XXXIV, 1920, p. 142-148, pl. XIII.

Johnston (S. J.). - Proc. I,in. Soc. New-South Wales, XXXVII, 1912, p. 285-362, pl. XIV-XLIII.

LeInY (J.). - Proc. Acud. Nat. Sci. Phila., V, 1851, p. 205.

Looss (A.). - Bibliot. Zoolog., VI, 1894, p. 56.

- Zoolog. Jahrb. Syst., XII, 1899, p. 605-607.

- Zool. Jahrb. Syst., XVI, 1902, p. 411.

LoschGE (F.). - Naturforscher, XXI, 1875, p. 10.

Pachingen (A.). - Anatomie d. Distomum cygnoides Klausenburg, 1883.

Pagens rechen (H.). - Trematoden \& Trematodenlarven, 1857, p. 44.

Rudolphi (C.). - Entozoorum sive vermium intestinalium, II, 1809, p. 367 ; Entozoorum synopsis, 1819, p. 96, 370.

Sevo (H.). - Dobutsu Gaku Zassi, XIX, 1901, p. 121.

Siebold (C.). - Arch. Naturgesch., I, 1835, p. 66.

Sivitzis (D.). - Contribution à l'histoire naturelle des Trématodes. Les Distomes des poissons et des grenouilles des environs de rarsovie, 1905, p. 1-210, pl. A.B. I-IV (en russe).

Stafromi (1.). - Zoolog. Jahrb. Syst., XVII, 1903, p. 411.

YaMaguTi (S.). - Iapanese Journ. Zool., VI, 1936, p. 563-565, fig. 18-19.

ZevenF (J.). - Erster Nachtrag zur Naturgeschichte der Eingeweidewïrmer von I.A.C. Gocze, J.eipzig, 1800 , p. 175. 